\title{
Solution of Seventh Order Boundary Value Problems using Adomian Decomposition Method
}

\author{
Shahid S. Siddiqi $\stackrel{*}{,}$ Muzammal Iftikhar ${ }^{\dagger}$
}

\begin{abstract}
Adomian decomposition method is used for solving the seventh order boundary value problems. The approximate solutions of the problems are calculated in the form of a rapid convergent series and not at grid points. Two numerical examples have been considered to illustrate the efficiency and implementation of the method.
\end{abstract}

Keywords: Adomian decomposition method; Seventh order boundary value problems; Linear and nonlinear problems; Series solution.

\section{Introduction}

The boundary value problems of ordinary differential equations play an important role in many fields. The theory of seventh order boundary value problems is seldom in the numerical analysis literature. These problems generally arise in modelling induction motors with two rotor circuits. The induction motor behavior is represented by a fifth order differential equation model. This model contains two stator state variables, two rotor state variables and one shaft speed. Normally, two more variables must be added to account for the effects of a second rotor circuit representing deep bars, a starting cage or rotor distributed parameters. To avoid the computational burden of additional state variables when additional rotor circuits are required, model is often limited to the fifth order and rotor impedance is algebraically altered as function of rotor speed. This is done under the assumption that the frequency of rotor currents depends on rotor speed. This approach is efficient for the steady state response with sinusoidal voltage, but it does not hold up during the transient conditions, when rotor frequency is not a single value. So, the behavior of such models show up in the seventh order boundary value problems [10].

Presently, the literature on the numerical solutions of seventh order boundary value problems and associated eigen value problems is seldom. Siddiqi and Ghazala [12, 13, 14, 15,

\footnotetext{
*Department of Mathematics, University of the Punjab, Lahore 54590, Pakistan. Email: shahidsiddiqiprof@yahoo.co.uk

${ }^{\dagger}$ Department of Mathematics, University of the Punjab, Lahore 54590, Pakistan. Email: miftikhar@hotmail.com
} 
[16, 17, 18] presented the solutions of fifth, sixth, eighth, tenth and twelfth order boundary value problems using polynomial and non-polynomial spline techniques. Siddiqi and Twizell [19, 20, 21] presented the solution of eighth, tenth and twelfth order boundary value problems using eighth, tenth and twelfth degree splines respectively.

The Adomian decomposition method was introduced and developed by George Adomian [1, 2, 3, 4]. This method has been applied to a wide class of linear and nonlinear ordinary differential equations, partial differential equations, integral equations and integro-differential equations [8, 9, 22, 23. Wazwaz [23, 24] provided the solution of fifth order and sixth order boundary value problems by the modified decomposition method. Convergence of Adomian decomposition method was studied by Cherruault et al. [ㅁ, 6, 7]. In this paper, the Adomian decomposition method [1, 2, 3, 4] is applied on the seventh order boundary value problem. An algorithm for approximate solution by Adomian method is developed for such problems. This method provides solutions in terms of a rapidly convergent series.

\section{Adomian Decomposition Method}

Consider the operator form of differential equation

$$
L u+R u+N u=f,
$$

where $L$ is the highest order derivative operator with the assumption that $L^{-1}$ exists, $R$ is a linear derivative operator of order less than $L, N u$ is the nonlinear term and $f$ is the source term. Applying the inverse operator $L^{-1}$ to both sides of Eq. (2.1 and using the given conditions yields the following

$$
u=g-L^{-1}(R u)-L^{-1}(N u),
$$

where the function $g$ represents the term obtained after integrating the source term $f$ and using the given conditions. The solution $u(x)$ can thus, be defined as

$$
u(x)=\sum_{n=0}^{\infty} u_{n}(x),
$$

where the components $u_{0}, u_{1}, u_{2}, \ldots$, are determined by the following recursive relation

$$
\begin{aligned}
& u_{0}(x)=g, \\
& u_{k+1}(x)=-L^{-1} R\left(u_{k}\right)-L^{-1} N\left(u_{k}\right), \quad k \geq 0 .
\end{aligned}
$$

The nonlinear term $N u$ is expressed in terms of an infinite series of the Adomian polynomials given by

$$
N u=\sum_{n=0}^{\infty} A_{n}
$$

where $A_{n}$ are Adomian polynomials [3] defined by

$$
A_{n}=\frac{1}{n !} \frac{d^{n}}{d p^{n}}\left[N\left(\sum_{k=0}^{\infty} p^{k} u_{k}\right)\right]_{p=0}, \quad k=0,1,2, \ldots
$$




\section{Analysis of the Method}

Consider the following seventh order boundary value problem

$$
u^{(7)}(x)=\phi(x) u+\psi(x)+f(x, u), \quad 0 \leq x \leq b,
$$

with boundary conditions

$$
\begin{aligned}
& u^{(i)}(0)=\alpha_{i}, \quad i=0,1,2,3, \\
& u^{(j)}(b)=\beta_{j}, \quad j=0,1,2 .
\end{aligned}
$$

where $\alpha_{i}, i=0,1,2,3$ and $\beta_{j}, j=0,1,2$ are finite real constants and the function $f$ is continuous on $[0, b]$.

The problem (3.1), in operator form, can be rewritten as

$$
L u=\phi(x) u+\psi(x)+f(x, u),
$$

where the derivative operator $L$ is given by

$$
L=\frac{d^{7}}{d x^{7}}
$$

The inverse operator $L^{-1}$ is therefore defined as

$$
L^{-1}(.)=\int_{0}^{x} \int_{0}^{x} \int_{0}^{x} \int_{0}^{x} \int_{0}^{x} \int_{0}^{x} \int_{0}^{x}(.) d x d x d x d x d x d x d x .
$$

Operating with $L^{-1}$ on Eq. 3.3 gives

$$
\begin{aligned}
u(x)= & \alpha_{0}+\alpha_{1} x+\frac{1}{2 !} \alpha_{2} x^{2}+\frac{1}{3 !} \alpha_{3} x^{3}+\frac{1}{4 !} \alpha_{4} x^{4}+\frac{1}{5 !} \alpha_{5} x^{5}+\frac{1}{6 !} \alpha_{6} x^{6} \\
& +L^{-1}(\phi(x) u)+L^{-1}(\psi(x))+L^{-1}(f(x, u)),
\end{aligned}
$$

using the boundary conditions (3.2) at $x=0$ yields

$$
\begin{aligned}
u(x)= & \alpha_{0}+\alpha_{1} x+\frac{1}{2 !} \alpha_{2} x^{2}+\frac{1}{3 !} \alpha_{3} x^{3}+\frac{1}{4 !} A x^{4}+\frac{1}{5 !} B x^{5}+\frac{1}{6 !} C x^{6}+ \\
& +L^{-1}(\phi(x) u)+L^{-1}(\psi(x))+L^{-1}(f(x, u)),
\end{aligned}
$$

where the constants

$$
A=u^{(4)}(0), \quad B=u^{(5)}(0), \quad C=u^{(6)}(0),
$$

will be determined using boundary conditions at $x=b$.

The Adomian method determines the solution $u(x)$ in terms of the following decomposition series

$$
u(x)=\sum_{n=0}^{\infty} u_{n}(x) .
$$


Substituting Eq. (3.9) into Eq. (3.7) leads to

$$
\begin{aligned}
\sum_{n=0}^{\infty} u_{n}(x)= & \alpha_{0}+\alpha_{1} x+\frac{1}{2 !} \alpha_{2} x^{2}+\frac{1}{3 !} \alpha_{3} x^{3}+\frac{1}{4 !} A x^{4}+\frac{1}{5 !} B x^{5}+\frac{1}{6 !} C x^{6}+ \\
& +L^{-1}\left(\phi(x) \sum_{n=0}^{\infty} u_{n}(x)\right)+L^{-1}(\psi(x))+L^{-1}(f(x, u)) .
\end{aligned}
$$

To determine the components $u_{n}(x), n \geq 0$, the following recurrence relation will be used

$$
\begin{aligned}
u_{0}(x) & =\alpha_{0}+\alpha_{1} x+\frac{1}{2 !} \alpha_{2} x^{2}+\frac{1}{3 !} \alpha_{3} x^{3}+\frac{1}{4 !} A x^{4}+\frac{1}{5 !} B x^{5}+\frac{1}{6 !} C x^{6}+L^{-1}(\psi(x)), \\
u_{k+1}(x) & =L^{-1}\left(\phi(x) \sum_{k=0}^{\infty} u_{k}(x)\right)+L^{-1}\left(\sum_{k=0}^{\infty} A_{k}\right), \quad k \geq 0,
\end{aligned}
$$

where the nonlinear function $f(x, u)$ can be decomposed into an infinite series of Adomian polynomials given by

$$
f(x, u)=\sum_{k=0}^{\infty} A_{k}
$$

where $A_{k}$ are Adomian polynomials $[3$ defined by

$$
A_{k}=\frac{1}{k !} \frac{d^{k}}{d p^{k}}\left[N\left(\sum_{j=0}^{\infty} p^{j} u_{j}\right)\right]_{p=0}, \quad n=0,1,2, \ldots
$$

Keeping in view Eq. 3.11 - Eq. 3.13), the components $u_{0}, u_{1}, u_{2}, \ldots$ are calculable. By the Adomian method the solution can be constructed as

$$
u=\lim _{n \rightarrow \infty} \varphi_{n},
$$

where the $n$-term approximant is defined by

$$
\varphi_{n}=\sum_{i=0}^{n-1} u_{i}
$$

Applying the boundary conditions at $x=b$ to the approximant $\varphi_{n}$. The resulting algebraic system in $A, B$ and $C$ can be solved to produce approximations to constants $A, B$ and $C$. Finally, the approximated solution of the seventh order boundary value problem follows. To implement the method, four numerical examples are considered in the following section.

\section{$4 \quad$ Numerical Examples}

Example 4.1 Consider the linear seventh order boundary value problem

$$
u^{(7)}(x)=x u(x)+e^{x}\left(x^{2}-2 x-6\right), \quad 0 \leq x \leq 1,
$$


subject to the boundary conditions

$$
\begin{aligned}
& u(0)=1, \quad u(1)=0, \\
& u^{(1)}(0)=0, \quad u^{(1)}(1)=-e, \\
& u^{(2)}(0)=-1, \quad u^{(2)}(1)=-2 e, \\
& u^{(3)}(0)=-2 .
\end{aligned}
$$

The exact solution of the problem (4.1) is

$$
u(x)=(1-x) e^{x} .
$$

The problem (4.1), in operator form, can be rewritten as

$$
L u=x u(x)+e^{x}\left(x^{2}-2 x-6\right),
$$

Operating with $L^{-1}$ on Eq. 4.3 and using the boundary conditions 4.2 at $x=0$ gives

$$
\begin{aligned}
u(x)= & -63-64 x-\frac{35}{2 !} x^{2}-4 x^{3}+\left(-\frac{1}{2}+\frac{A}{24}\right) x^{4}+\left(-\frac{1}{30}+\frac{B}{120}\right) B x^{5}+\frac{1}{360}(2+C) x^{6} \\
& +e^{x}(-8+x)^{2}+L^{-1}(x u(x)),
\end{aligned}
$$

where the constants

$$
A=u^{(4)}(0), B=u^{(5)}(0), C=u^{(6)}(0),
$$

are to be determined. Substituting the decomposition series (2.3 for $u(x)$ in Eq. 4.4 ) yields

$$
\begin{aligned}
\sum_{n=0}^{\infty} u_{n}(x)= & -63-64 x-\frac{35}{2 !} x^{2}-4 x^{3}+\left(-\frac{1}{2}+\frac{A}{24}\right) x^{4}+\left(-\frac{1}{30}+\frac{B}{120}\right) B x^{5}+\frac{1}{360}(2+C) x^{6} \\
& +e^{x}(-8+x)^{2}+L^{-1}\left(x \sum_{n=0}^{\infty} u_{n}(x)\right) .
\end{aligned}
$$

Using the recurrence algorithm (3.11) the following relations are obtained

$$
\begin{aligned}
u_{0}(x)= & -63-64 x-\frac{35}{2 !} x^{2}-4 x^{3}+\left(-\frac{1}{2}+\frac{A}{24}\right) x^{4}+\left(-\frac{1}{30}+\frac{B}{120}\right) B x^{5}+\frac{1}{360}(2+C) x^{6} \\
& +e^{x}(-8+x)^{2}, \\
u_{k+1}(x)= & L^{-1}\left(x u_{k}(x)\right), \quad k \geq 0 .
\end{aligned}
$$

From Eq. 4.7 ,

$$
\begin{aligned}
u_{0}(x)= & -63-64 x-\frac{35}{2 !} x^{2}-4 x^{3}+\left(-\frac{1}{2}+\frac{A}{24}\right) x^{4}+\left(-\frac{1}{30}+\frac{B}{120}\right) B x^{5}+\frac{1}{360}(2+C) x^{6} \\
& +e^{x}(-8+x)^{2}, \\
u_{1}(x)= & L^{-1}\left(x u_{0}(x)\right), \\
= & 1848+1392 x+505 x^{2}+116 x^{3}+\frac{37}{2} x^{4}+\frac{31}{15} x^{5}+\frac{17}{120} x^{6}-\frac{x^{8}}{640}-\frac{x^{9}}{3780}-\frac{x^{10}}{34560} \\
& -\frac{x^{11}}{415800}+\frac{(-12+A) x^{12}}{95800320}+\frac{(-4+B) x^{13}}{1037836800}+\frac{(2+C) x^{14}}{12454041600}+e^{x}(-1848+456 x \\
& \left.-37 x^{2}+x^{3}\right),
\end{aligned}
$$


The series solution of $u(x)$ is found to be as an approximation with four components

$$
u(x)=u_{0}(x)+u_{1}(x)+u_{2}(x)+u_{3}(x) .
$$

The unknown constants $A, B$ and $C$ can be obtained by imposing the boundary conditions at $x=1$ on the four-term approximant $\varphi_{4}$ defined by Eq. 4. 4 , as

$$
A=-3.0000001004600083, \quad B=-3.9999991664171546, \quad C=-5.000002140841064 .
$$

Finally, the series solution can be written as

$$
\begin{aligned}
u(x)= & 1-\frac{x^{2}}{2}-\frac{x^{3}}{3}-0.125 x^{4}-0.0333333 x^{5}-0.00694445 x^{6}-\frac{x^{7}}{840}-\frac{x^{8}}{5760}-\frac{x^{9}}{45360} \\
& -\frac{x^{10}}{403200}-\frac{x^{11}}{3991680}-\left(2.29644 \times 10^{-8}\right) x^{12}-\left(1.60591 \times 10^{-10}\right) x^{13} \\
& +O\left(x^{14}\right) .
\end{aligned}
$$

The comparison of the exact solution with the series solution of the problem (4.1) is given in Table 1, which shows that the method is quite efficient. Figure 1 also endorses the efficiency of the method.

Example 4.2 Consider the following seventh order nonlinear boundary value problem

$$
u^{(7)}(x)=-e^{x} u^{2}(x), \quad 0<x<1,
$$

subject to the boundary conditions

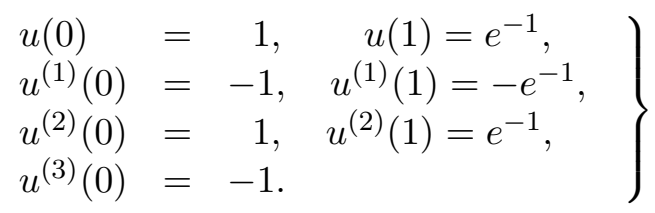

The exact solution of the problem (4.2) is

$$
u(x)=e^{-x} .
$$

The problem 4.11), in operator form, can be rewritten as

$$
L u=e^{x} u^{2}(x),
$$

Operating with $L^{-1}$ on Eq. 4.13 and using the boundary conditions 4.12 at $x=0$ gives

$$
u(x)=1-x+\frac{1}{2 !} x^{2}-\frac{1}{3 !} x^{3}+\frac{1}{4 !} A x^{4}+\frac{1}{5 !} B x^{5}+\frac{1}{6 !} C x^{6}+L^{-1}\left(-e^{x} u^{2}(x)\right),
$$

where the constants

$$
A=u^{(4)}(0), B=u^{(5)}(0), C=u^{(6)}(0)
$$




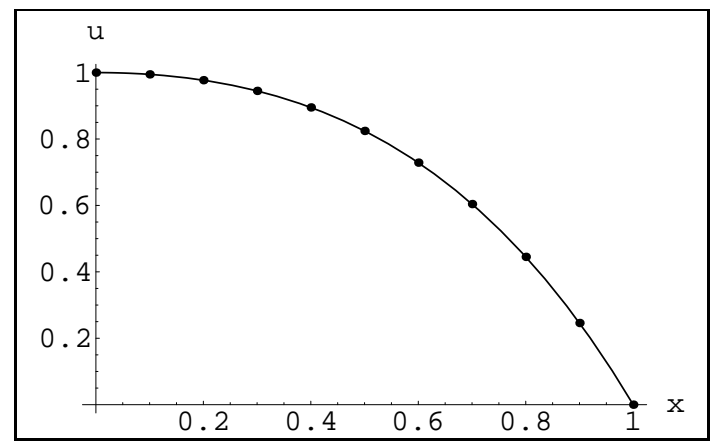

Figure 1: Comparison of the approximate solution with the exact solution for the problem (4.1). Dotted line: approximate solution, solid line: the exact solution.

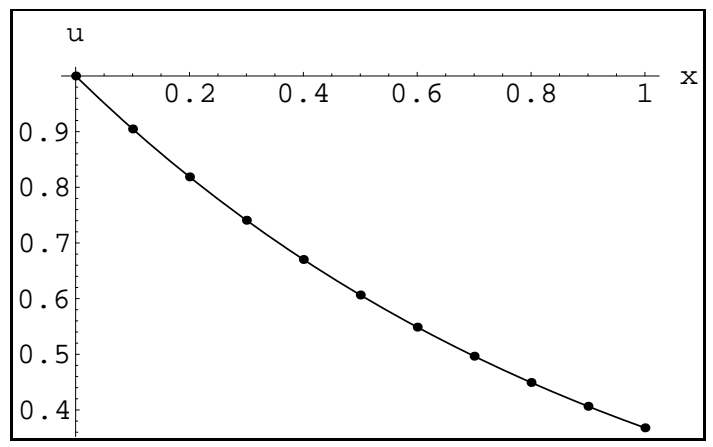

Figure 2: Comparison of the approximate solution with the exact solution for the problem (4.2). Dotted line: approximate solution, solid line: the exact solution.

are to be determined. Substituting the decomposition series 2.3 for $u(x)$ and the series of polynomials 2.5 for $u^{2}(x)$ in Eq. 4.14 yields

$$
\begin{aligned}
\sum_{n=0}^{\infty} u_{n}(x)= & 1-x+\frac{1}{2 !} x^{2}-\frac{1}{3 !} x^{3}+\frac{1}{4 !} A x^{4}+\frac{1}{5 !} B x^{5}+\frac{1}{6 !} C x^{6} \\
& +L^{-1}\left(-e^{x} \sum_{n=0}^{\infty} A_{n}\right),
\end{aligned}
$$

where $A_{n}$ are the Adomian polynomials for the nonlinear term $N u=u^{2}(x)$, thus given by

$$
\begin{aligned}
A_{0} & =N\left(u_{0}\right)=u_{0}^{2}(x) \\
A_{1} & =u_{0}(x) N^{\prime}\left(u_{0}\right)=2 u_{0}(x) u_{1}(x) \\
A_{2} & =u_{2}(x) N^{\prime}\left(u_{0}\right)+\frac{u_{1}^{2}(x)}{2 !} N^{\prime \prime}\left(u_{0}\right)=2 u_{0}(x) u_{2}(x)+u_{1}^{2}(x), \\
& \vdots
\end{aligned}
$$

Using the recurrence algorithm (3.11) yields

$$
\begin{aligned}
u_{0}(x) & =1-x+\frac{1}{2 !} x^{2}-\frac{1}{3 !} x^{3}+\frac{1}{4 !} A x^{4}+\frac{1}{5 !} B x^{5}+\frac{1}{6 !} C x^{6}, \\
u_{k+1}(x) & =L^{-1}\left(-e^{x} A_{k}\right), \quad k \geq 0,
\end{aligned}
$$

From 4.17 and (4.18),

$$
\begin{aligned}
u_{0}(x)= & 1-x+\frac{1}{2 !} x^{2}-\frac{1}{3 !} x^{3}+\frac{1}{4 !} A x^{4}+\frac{1}{5 !} B x^{5}+\frac{1}{6 !} C x^{6}, \\
u_{1}(x)= & L^{-1}\left(-e^{x} A_{0}\right), \\
= & -\frac{x^{7}}{5040}+\frac{x^{8}}{40320}-\frac{x^{9}}{362880}+\frac{x^{10}}{3628800}+\left(\frac{1}{39916800}-\frac{A}{19958400}\right) x^{11} \\
& -\left(\frac{1}{479001600}+\frac{B}{239500800}\right) x^{12}+\left(\frac{1}{6227020800}-\frac{C}{3113510400}\right) x^{13}+O\left(x^{14}\right),
\end{aligned}
$$




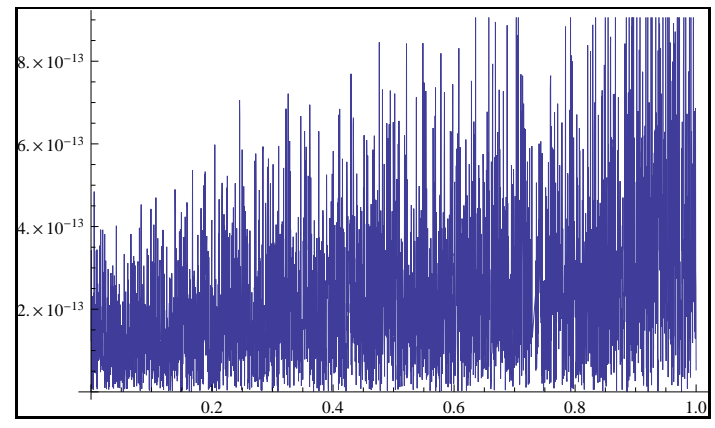

Figure 3: Absolute Error for the problem (4.3).

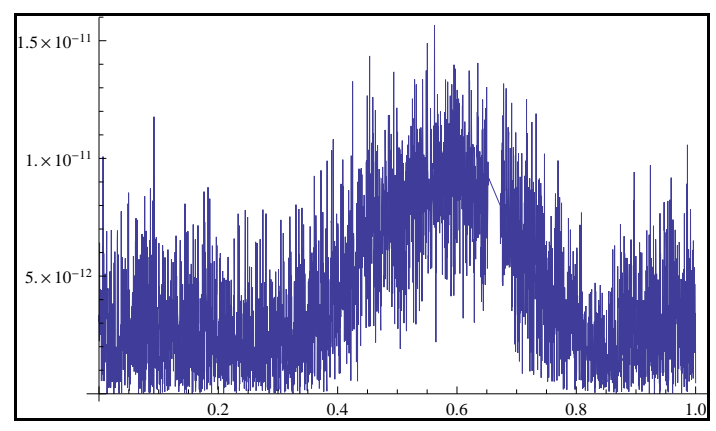

Figure 4: Absolute Error for the problem (4.4).

The series solution of $u(x)$ is found to be as an approximation with two components

$$
u(x)=u_{0}(x)+u_{1}(x) .
$$

The unknown constant $A, B$, and $C$ can be obtained by imposing the boundary conditions at $x=1$ on the two-term approximant $\varphi_{2}$ defined by Eq. 4.19 as

$$
A=1.0000000197456873, \quad B=-1.000000228700079, \quad C=1.0000008112153262 .
$$

Finally, the series solution can be written as

$$
\begin{aligned}
u(x)= & 1-x+0.5 x^{2}-0.166667 x^{3}+0.0416667 x^{4}-0.00833334 x^{5}+0.00138889 x^{6} \\
& -0.000198413 x^{7}+0.0000248016 x^{8}-\left(2.75573 \times 10^{-6}\right) x^{9}+\left(2.75573 \times 10^{-7}\right) x^{10} \\
& -\left(2.50521 \times 10^{-8}\right) x^{11}+\left(2.08768 \times 10^{-9}\right) x^{12}-\left(1.60591 \times 10^{-10}\right) x^{13} \\
& +O\left(x^{14}\right) .
\end{aligned}
$$

The comparison of the exact solution with the solution of the problem (4.2) is given in Table 2 , which shows that the method is quite efficient. Figure 2 also endorses the efficiency of the method.

Example 4.3 Consider the following seventh order linear boundary value problem

$$
\begin{aligned}
u^{(7)}(x) & =-u(x)-e^{x}\left(35+12 x+2 x^{2}\right), \quad 0 \leq x \leq 1, \\
u(0) & =0, \quad u(1)=0 \\
u^{(1)}(0) & =1, \quad u^{(1)}(1)=-e, \\
u^{(2)}(0) & =0, \quad u^{(2)}(1)=-4 e, \\
u^{(3)}(0) & =-3 .
\end{aligned}
$$

The exact solution of the Example 3.1 is $u(x)=x(1-x) e^{x}$ [11].

Following the procedure of the Example 4.1, this problem is solved. It is observed that the errors in absolute values are better than those of Siddiqi and Iftikhar [11] as shown in Table 3. In Figure 3 absolute errors are plotted. 
Example 4.4 The following seventh order nonlinear boundary value problem is considered

$$
\left.\begin{array}{rl}
u^{(7)}(x) & =u(x) u^{\prime}(x)+e^{-2 x}\left(2+e^{x}(x-8)-3 x+x^{2}\right), \quad 0 \leq x \leq 1, \\
u(0) & =1, \quad u(1)=0 \\
u^{(1)}(0) & =-2, u^{(1)}(1)=-1 / e \\
u^{(2)}(0) & =3, u^{(2)}(1)=2 / e \\
u^{(3)}(0) & =-4
\end{array}\right\}
$$

The exact solution of the Example 3.2 is $u(x)=(1-x) e^{-x}$.

Following the procedure of the previous problem 4.2, this problem is solved. The comparison of the exact solution with the solution of the problem 4.2 is given in Table 4, which shows that the method is quite efficient. In Figure 4 absolute errors are plotted.

Conclusion In this paper, the Adomian decomposition method has been applied to obtain the numerical solutions of linear and nonlinear seventh order boundary value problems. The numerical results show that the method is quite efficient for solving high order boundary value problems arising in various fields of engineering and science.

Table 1: Comparison of numerical results for the problem 4.1

\begin{tabular}{|c|c|c|c|}
\hline$x$ & Exact solution & Approximate series solution & Absolute Error \\
\hline 0.0 & 1.0000 & 1.0000 & 0.0000 \\
\hline 0.1 & 0.0994 & 0.0994 & $4.3972 \mathrm{E}-10$ \\
\hline 0.2 & 0.9771 & 0.9771 & $4.9251 \mathrm{E}-10$ \\
\hline 0.3 & 0.9449 & 0.9449 & $7.4067 \mathrm{E}-10$ \\
\hline 0.4 & 0.8950 & 0.8950 & $6.6537 \mathrm{E}-10$ \\
\hline 0.5 & 0.8243 & 0.8243 & $3.0059 \mathrm{E}-11$ \\
\hline 0.6 & 0.7288 & 0.7288 & $4.3591 \mathrm{E}-10$ \\
\hline 0.7 & 0.6041 & 0.6041 & $3.6735 \mathrm{E}-10$ \\
\hline 0.8 & 0.4451 & 0.4451 & $7.2753 \mathrm{E}-10$ \\
\hline 0.9 & 0.2459 & 0.2459 & $7.0036 \mathrm{E}-10$ \\
\hline 1.0 & 0.0000 & $2.2191 \mathrm{E}-10$ & $2.2191 \mathrm{E}-10$ \\
\hline
\end{tabular}

Table 2: Comparison of numerical results for problem 4.2

\begin{tabular}{|c|c|c|c|}
\hline$x$ & Exact solution & Approximate series solution & Absolute Error \\
\hline 0.0 & 1.0000 & 1.0000 & 0.0000 \\
\hline 0.1 & 0.9048 & 0.9048 & $1.5676 \mathrm{E}-9$ \\
\hline 0.2 & 0.8187 & 0.8187 & $1.6418 \mathrm{E}-9$ \\
\hline 0.3 & 0.7408 & 0.7408 & $4.9680 \mathrm{E}-9$ \\
\hline 0.4 & 0.6703 & 0.6703 & $1.5514 \mathrm{E}-9$ \\
\hline 0.5 & 0.6065 & 0.6065 & $1.5274 \mathrm{E}-9$ \\
\hline 0.6 & 0.5488 & 0.5488 & $2.4958 \mathrm{E}-9$ \\
\hline 0.7 & 0.4965 & 0.4965 & $1.3993 \mathrm{E}-8$ \\
\hline 0.8 & 0.4493 & 0.4493 & $2.5593 \mathrm{E}-9$ \\
\hline 0.9 & 0.4065 & 0.4065 & $5.4089 \mathrm{E}-9$ \\
\hline 1.0 & 0.3678 & 0.3678 & $1.1034 \mathrm{E}-9$ \\
\hline
\end{tabular}


Table 3: Comparison of numerical results for the problem 4.3

\begin{tabular}{|c|c|c|c|c|}
\hline$x$ & $\begin{array}{c}\text { Exact } \\
\text { solution }\end{array}$ & $\begin{array}{c}\text { Approximate } \\
\text { series solution }\end{array}$ & $\begin{array}{c}\text { Absolute Error } \\
\text { present method }\end{array}$ & $\begin{array}{c}\text { Absolute Error } \\
\text { Siddiqi and Iftikhar [1] }\end{array}$ \\
\hline 0.0 & 0.0000 & 0.0000 & 0.0000 & 0.0000 \\
\hline 0.1 & 0.9946 & 0.9946 & $1.23082 \mathrm{E}-13$ & $8.55607 \mathrm{E}-13$ \\
\hline 0.2 & 0.1954 & 0.1954 & $3.7792 \mathrm{E}-13$ & $9.94041 \mathrm{E}-12$ \\
\hline 0.3 & 0.2835 & 0.2835 & $2.37421 \mathrm{E}-13$ & $3.52244 \mathrm{E}-11$ \\
\hline 0.4 & 0.3580 & 0.3580 & $3.62099 \mathrm{E}-13$ & $7.3224 \mathrm{E}-10$ \\
\hline 0.5 & 0.4122 & 0.4122 & $9.39249 \mathrm{E}-14$ & $1.08769 \mathrm{E}-10$ \\
\hline 0.6 & 0.4373 & 0.4373 & $4.82947 \mathrm{E}-13$ & $1.29035 \mathrm{E}-10$ \\
\hline 0.7 & 0.4229 & 0.4229 & $1.09135 \mathrm{E}-13$ & $1.51466 \mathrm{E}-10$ \\
\hline 0.8 & 0.3561 & 0.3561 & $1.64868 \mathrm{E}-14$ & $2.717974 \mathrm{E}-10$ \\
\hline 0.9 & 0.2214 & 0.2214 & $7.25975 \mathrm{E}-13$ & $7.48179 \mathrm{E}-10$ \\
\hline 1.0 & 0.0000 & $-4.54747 \mathrm{E}-13$ & $4.54747 \mathrm{E}-13$ & $2.1729 \mathrm{E}-09$ \\
\hline
\end{tabular}

Table 4: Comparison of numerical results for the problem 4.4

\begin{tabular}{|c|c|c|c|}
\hline$x$ & Exact solution & Approximate series solution & Absolute Error \\
\hline 0.0 & 1.0000 & 1.0000 & $1.67932 \mathrm{E}-12$ \\
\hline 0.1 & 0.814354 & 1.1051 & $2.96696 \mathrm{E}-12$ \\
\hline 0.2 & 0.654985 & 0.654985 & $1.26055 \mathrm{E}-12$ \\
\hline 0.3 & 0.518573 & 0.518573 & $2.10898 \mathrm{E}-12$ \\
\hline 0.4 & 0.402192 & 0.402192 & $6.68926 \mathrm{E}-12$ \\
\hline 0.5 & 0.303265 & 0.303265 & $7.21923 \mathrm{E}-12$ \\
\hline 0.6 & 0.219525 & 0.219525 & $9.75339 \mathrm{E}-12$ \\
\hline 0.7 & 0.148976 & 0.148976 & $2.19552 \mathrm{E}-12$ \\
\hline 0.8 & 0.0898658 & 0.0898658 & $4.24917 \mathrm{E}-12$ \\
\hline 0.9 & 0.040657 & 0.040657 & $2.27311 \mathrm{E}-13$ \\
\hline 1.0 & 0.0000 & $4.42298 \mathrm{E}-12$ & $4.42298 \mathrm{E}-12$ \\
\hline
\end{tabular}

\section{References}

[1] G. Adomian, Nonlinear stochastic operator equations, Academic Press, San Diego (1986).

[2] _ A review of the decomposition method in applied mathematics, J. Math. Anal. Appl. 135) (1988), 501-544.

[3] _ Solving frontier problems of physics: The decomposition method, Kluwer, Boston (1994).

[4] G. Adomian and R. Rach, Analytic solution of nonlinear boundary-value problems in several dimensions by decomposition, J. Math. Anal. Appl. 174 (1993), 118-137.

[5] Y. Cherrauault, G. Saccomandi, and B. Some, New results for convergence of adomians method applied to integral equations, Math. Comput. Modelling 16 (2) (1992), 85-93.

[6] Y. Cherruault, Convergence of adomians method, Math. Comput. Modelling 14 (1990), 83-86. 
[7] Y. Cherruault and G. Adomian, Decomposition method: a new proof of convergence, Math. Comput. Modelling 18 (12) (1993), 103-106.

[8] M. Dehghan and F. Shakeri, The numerical solution of the second painleve equation, Num. Meth. PDEs 25 (2009), 1238-1259.

[9] R. Rach, On the adomian decomposition method and comparisons with picards method, J. Math. Anal. Appl. 128 (1987), 480-483.

[10] G. Richards and P. R. R. Sarma, Reduced order models for induction motors with two rotor circuits, IEEE Transactions on Energy Conversion 9(4) (1994), 673-678.

[11] S. S. Siddiqi and M. Iftikhar, Solution of seventh order boundary value problems by variation of parameters method, Res. J. Appl. Sci., Engin. Tech. 5(1) (2013), 176-179.

[12] Shahid S. Siddiqi and Ghazala Akram, Solutions of fifth order boundary-value problems using nonpolynomial spline technique, Appl. Math. Comput. 175(2) (2006), 1574-1581.

[13] _ Solutions of sixth order boundary-value problems using nonpolynomial spline technique, Appl. Math. Comput. 181 (2006), 708-720.

[14] Solutions of twelfth-order boundary value problems using thirteen degree spline, Appl. Math. Comput. 182 (2006), 1443-1453.

[15] _ Solution of eighth order boundary value problems using the non-polynomial spline techniques, Int. J. Comput. Math. 84 (2007), 347-368.

[16] _ Solutions of 10th-order boundary value problems using non-polynomial spline technique, Appl. Math. Comput. 190 (2007), 641-651.

[17] _ Solutions of tenth-order boundary value problems using eleventh degree spline, Appl. Math. Comput. 185 (2007), 115-127.

[18] $ـ$ Solutions of 12th-order boundary value problems using non-polynomial spline technique, Appl. Math. Comput. 199 (2) (2008), 559-871.

[19] Shahid S. Siddiqi and E. H. Twizell, Spline solutions of linear tenth-order boundary value problems, Int. J. Comput. Math. 68 (1996), 345-362.

[20] _ Spline solutions of linear twelfth-order boundary value problems, J. Comput. Appl. Math 78 (1997), 371-390.

[21] _ Spline solutions of linear eighth-order boundary value problems, Comput. Meth. Appl. Mech. Eng 131 (1998), 309-325.

[22] A.M. Wazwaz, A comparison between adomian decomposition method and taylor series method in the series solutions, Appl. Math. Comput. 79 (1998), 37-44.

[23] _ The numerical solution of fifth-order boundary value problems by the decomposition method, J. Comput. Appl. Math. 136 (2001), 259-270. 
[24] - The numerical solution of sixth-order boundary value problems by the modified decomposition method, Appl. Math. Comput. 118 (2001), 311-325. 